\title{
COVID-19 and Stigma: Evolution of Self-restraint Behavior
}

\author{
Kenichi Kurita ${ }^{1}$ (D) Shunsuke Managi ${ }^{1}$
}

Accepted: 3 January 2022 / Published online: 25 January 2022

(c) The Author(s), under exclusive licence to Springer Science+Business Media, LLC, part of Springer Nature 2022

\begin{abstract}
Social stigma can effectively prevent people from going out and possibly spreading COVID19. Using the framework of replicator dynamics, we analyze the interaction between selfrestraint behavior, infection with viruses such as COVID-19, and stigma against going out. Our model is analytically solvable with respect to an interior steady state in contrast to the previous model of COVID-19 with stigma. We show that a non-legally binding policy reduces the number of people going out in a steady state.
\end{abstract}

Keywords COVID-19 $\cdot$ Stigma $\cdot$ Self-restraint behavior $\cdot$ Non-pharmaceutical interventions $\cdot$ Replicator dynamics

\section{Introduction}

Countries around the world are implementing various policies to control the spread of COVID-19 through trial and error. Specifically, governments are implementing policies to reduce the chance of contact with the disease to reduce the rate of infection. The following two types of policies restrict behavior to prevent the spread of infectious diseases: legally enforceable behavioral restrictions with fines or punishments and non-legally binding behavioral restrictions based on individual self-restraint without penalties.

Policies enacted by several European countries and the USA have implemented legally enforceable behavioral restrictions. The USA has the highest number of cases worldwide as of October 2, 2020, with 7.4 million infected and 211,000 dead [38]. New York State, which declared a state of emergency on March 7, mandated in principle 100\% telecommuting

This article is part of the topical collection "Modeling and Control of Epidemics" edited by Quanyan Zhu, Elena Gubar and Eitan Altman.

The authors acknowledge the support from JSPS KAKENHI Grant Numbers JP19K23194 and JP20K13486, Research Institute for Humanity and Nature (Grant No. Feasibility Project 14200138).

Kenichi Kurita

kurita.kenichi.564@m.kyushu-u.ac.jp

Shunsuke Managi

managi@doc.kyushu-u.ac.jp

1 Urban Institute and Department of Civil Engineering, School of Engineering, Kyushu University, 744

Motooka, Nishi-ku, Fukuoka 819-0395, Japan 
starting March 22, on the governor's order. Companies can be fined up to 10,000 US dollars if they do not follow through and cause severe physical harm to their employees. The state of public health emergency imposed in France allows the Prime Minister, with the advice of the Minister of Health, to immediately implement a series of restrictive measures applicable throughout the country, which is a legally binding policy [13]. Individuals who go out for purposes other than those authorized by the government, such as the purchase of living essentials, are fined between 135 and 3700 Euros. In Italy, where the number of COVID-19related deaths is at 35,968 as of October 2, 2020 [9], a decree was passed on March 10, 2020, imposing a nationwide curfew, with penalties of up to 3000 Euros for those who do not carry a "certificate" stating the place and reason they had to go out. In Spain, Prime Minister Pedro Sánchez ordered a "state of alarm," which was legally binding on March 14 [4]. Under the Spanish state of emergency, breachers were arrested or fined between 601 and 30,000 Euros.

In contrast, some countries, such as Japan and Sweden, imposed a non-legally binding policy based on individual self-restraint without enforcement. In Japan, the government declared a state of emergency, which is not legally binding, which significantly restrained people from going out $[21,25,39]$. It is widely considered to have been more successful in controlling the number of infections than in other OECD countries [20,28]. How many people in Japan refrain from going out under the non-binding declaration of a state of emergency? To answer this question, we consider the interaction between infection risk, stigma, and the player's decision-making. In Japan, the phenomenon of a "self-restraint police" (Jishuku Keisatsu in Japanese) emerged under the state of emergency. The "self-restraint police" is a colloquial term for ordinary citizens who crack down on or attack individuals or shops that do not respond to government requests to refrain from going out or doing business. They have posted expletives on the doors of restaurants open for business and scratched cars with outof-prefecture plates $[40,41]$. The self-restraint police symbolize the stigma against those who do not comply with requests for self-restraint. This suggests that even unenforceable policies can discourage people from going out to avoid social stigma. We apply an evolutionary game to analyze self-restraint behavior in the context of infectious disease epidemics from a stigmatization perspective.

Research on stigma has evolved around social psychology [29] and sociology, beginning with the discussion by Goffman [16]. For example, Lieberman et al. [26] explored whether the relation between disgust sensitivity and stigma of obesity is specific to pathogen disgust or whether sexual and moral disgust is also associated with negative attitudes toward obese people. There are also several studies on stigma in economics, Moffitt [32], Besley and Coate [5], Bhargava and Manoli [6] study welfare stigma [19,24,27], Rasmusen [34] analyzes the stigma related to criminal record, Kim [22] analyzes the stigma against tax evasion, and Ennis and Weinberg [11] investigate financial stigma. It is important to analyze stigma in terms of going-out behavior during an infectious disease epidemic, as it may play a similar role in fear of infection. We suppose that the psychological costs of stigma intensify under a declared state of emergency in the model. Consequently, we show that the number of people going out in a steady state under the declared state of emergency is less than the number without it.

Several empirical studies analyze the effect of Japan's non-legally enforceable emergency declarations $[21,23,46]$. Kobayashi et al. [23] show that the declaration and extension of the state of emergency have achieved some success in controlling the COVID-19 pandemic. Other studies analyze the effect of a legally binding lockdown on the economy $[1,3,10$, 12,15,18,30,31]. Acemoglu et al. [1] and Alvarez et al. [3] discuss the optimal lockdown policy. Mandel and Veetil [30] estimate the costs of a lockdown in some sectors of the global economy using a multi-sector model. Bouveret and Mandel [7] investigate the confinement 
of epidemic spread in networks from a normative perspective considering the interactions between individual behavior, network structure and social efficiency.

We present an investigation of the evolutionary model, specifically, the replicator dynamics of self-restraint behavior when stigma and the risk of infection change with the number of players going out. Evolutionary game and replicator dynamics are widely studied and applied in economics $[2,8,14,17,33,35-37,42-45,47]$. Taylor and Jonker [37] was the first to model replicator dynamics, which has since been applied in many fields and for various issues. For instance, Safarzyńnska and Van den Bergh [35] analyzed technological change using replicator dynamics, Cerqueti et al. [8] and Shi et al. [36] consider a dynamic perspective of economic interactions and social tolerance applying it, and Itaya and Kurita [19] analyze the replicator dynamics of taking-up behavior in welfare programs.

Although the number of studies on COVID-19 is increasing, few studies consider stigma. One of the few exceptions is Katafuchi et al. [21]. They analyzed the theoretical model with stigma and infection risk and empirically tested the theoretical results using mobility data. However, they consider infection risk as exogenous, and this assumption is strict. Moreover, their model defines the fixed point of the number of players going out as an equilibrium point. This means that all players are rational enough to calculate each payoff and expect the number of players going out at least in equilibrium. Furthermore, their model is not analytically solvable for an interior solution. Finally, they analyze the static model; however, the pandemic changes drastically change over time.

This study contributes in the following ways. First, we endogenize both stigma cost and infection risk and weaken the rationality that players attain equilibrium using replicator dynamics to beyond concerns in the previous research mentioned here. Second, we present an analytically solvable model in contrast to the previous model of COVID-19 and stigma. Third, we show that the state of emergency has an effect on players' self-restraint behavior in a steady state. Forth, the social welfare analysis indicates that the number of players going out is larger than the socially optimal level without/under the state of emergency.

This paper proceeds as follows: In Sects. 2 and 3, we present the basic setting of the model and the replicator dynamics. Section 4 investigates whether the non-legally binding policy induces self-restraint behavior. Section 5 presents the results of the comparative statics. Section 6 includes the welfare analysis. Finally, Sect. 7 concludes.

\section{The Model}

We consider an economy with a population that is constant in time. There are two actions or strategy types: Going-out and Staying-home. Let $x(t)$ be the share of going-out players in the total population at time $t . \pi_{a}$ corresponds to player's payoff when player's action is $a$ where $a \in\{G, S\}, G$ is an abbreviation for "Going out" and $S$ is for "Staying home." Each payoff, $\pi_{G}$ and $\pi_{S}$, is set as follows:

$$
\begin{aligned}
\pi_{G} & =u_{\text {out }}-\gamma(x) c-\sigma s(x), \\
\pi_{S} & =u_{\text {home }} .
\end{aligned}
$$

Here, $x$ is the proportion of players going out to the total population, $u_{\text {out }}$ is the utility from going out, $u_{\text {home }}$ is the utility from staying home, $\gamma(x) c$ is the subjective expected cost of infection with the virus, $\gamma(x)$ is the subjective probability of infection with the virus, $c$ is the cost of infection with the virus, $\sigma s(x)$ is the stigma cost of going out, $\sigma$ is the relative size of stigma cost to infection cost, $s(x)$ is the stigma cost function. For analytical simplicity, we 
assume that the subjective probability of infection with the virus is an increasing function with the proportion of players going out in the total population as follows:

$$
\gamma(x)=\eta x,
$$

where $\eta(>0)$ is the parameter indicating the degree of increase in the subjective probability of infection of more people going out. Moreover, we assume that the stigma cost is a decreasing function with the proportion of players going out in the total population as follows:

$$
s(x)=\zeta_{0}-\zeta_{1} x,
$$

where $\zeta_{0}(>0)$ is the fixed stigma cost, $\zeta_{1} x$ is the flexible stigma cost, and $\zeta_{1}(>0)$ is the degree of stigma reduction of more people going out. This formulation of stigma cost is based on Lindbeck et al. [27] and Katafuchi et al. [21]. We assume that $s(1)=\zeta_{0}-\zeta_{1}>0$. This assumption means that the lowest level of stigma cost is not zero and positive.

\section{Replicator Dynamics}

Next, we show the replicator dynamics of the population share of players going out in the model. To achieve this, we need to check the expected payoff of each strategy.

We model the replicator dynamics of the going-out share in the total population by the following differential equation:

$$
\dot{x}=x(1-x)\left(\pi_{G}-\pi_{S}\right) .
$$

Substituting Eqs. (1) and (2) into Eq. (6), we can transform Eq. (5) as follows:

$$
\dot{x}=x(1-x)\left[u_{\text {out }}-u_{\text {home }}-\gamma(x) c-\sigma s(x)\right] .
$$

We derive the stationary point in the dynamics by solving (6), $\dot{x}=0$, as follows:

$$
x^{*} \in\{0, \hat{x}, 1\},
$$

where

$$
\hat{x}=\frac{u_{\mathrm{out}}-u_{\mathrm{home}}-\sigma \zeta_{0}}{\eta c-\sigma \zeta_{1}} .
$$

The condition for the interior stationary point is given, as shown in Lemma 1.

Lemma 1 The necessary and sufficient condition for $\hat{x} \in(0,1)$, is given by

$$
\sigma \zeta_{0}<u_{\text {out }}-u_{\text {home }}<\eta c+\sigma\left(\zeta_{0}-\zeta_{1}\right)
$$

Proof See "Appendix."

The stability analysis presents us with the following results:

Proposition 1 The interior stationary point $x^{*}=\hat{x}$ is asymptotically stable and $x^{*} \in\{0,1\}$ is unstable under the following condition:

$$
\eta c>\sigma \zeta_{1}
$$

\section{Proof See "Appendix."}

Proposition 1 suggests that the interior steady state, $\hat{x}$, is asymptotically stable when it exists. Figure 1 shows the dynamics of the population share of players going out and stationary points. There are three stationary points, $x^{*} \in\{0, \hat{x}, 1\}$. We can confirm that $x^{*}=\hat{x}$ is asymptotically stable and $x^{*} \in\{0,1\}$ is unstable, as shown in Fig. 1 . 


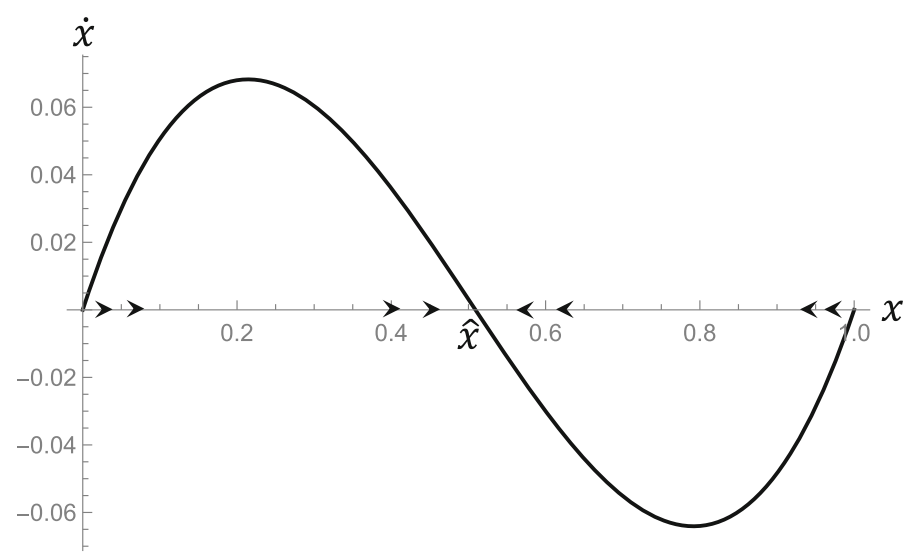

Fig. 1 Steady states without the state of emergency. Notes: The figure shows the evolution of $x$ with parameters as follows: $u_{\text {out }}=1, u_{\text {home }}=0.05, \eta=1, c=1.5, \sigma=0.5, \zeta_{0}=0.5$, and $\zeta_{1}=0.25$

\section{Effect of the Non-legally Binding State of Emergency}

Our aim is to investigate the effect of the non-legally binding policy on the stationary point. We introduce the policy variable $\iota \in\{0,1\}$ as follows:

$$
\pi_{G}=u_{\text {out }}-\gamma(x) c-(1+\rho \iota) \sigma s(x),
$$

where $\iota$ is the indicator variable of the state of emergency and $\rho>0$ is a parameter that expresses the amplification of stigma by the state of emergency. Therefore, this setting implies that stigma costs are enhanced by $(1+\rho)$ times more under the state of emergency than they would otherwise be. Let $\hat{x}_{1}$ denote the interior stationary point under the state of emergency and $\hat{x}_{0}$ without the state of emergency. $\hat{x}_{0}$ is equal to the right-hand side of (8) because $\hat{x}=\hat{x}_{0}$. The stationary points without the state of emergency are given as follows:

$$
x^{*} \in\left\{0, \hat{x}_{0}, 1\right\},
$$

where

$$
\hat{x}_{0}=\frac{u_{\mathrm{out}}-u_{\mathrm{home}}-\sigma \zeta_{0}}{\eta c-\sigma \zeta_{1}} .
$$

We can derive the stationary point under the state of emergency as follows:

$$
x^{*}=\left\{0, \hat{x}_{1}, 1\right\},
$$

where

$$
\hat{x}_{1}=\frac{u_{\text {out }}-u_{\text {home }}-(1+\rho) \sigma \zeta_{0}}{\eta c-(1+\rho) \sigma \zeta_{1}} .
$$

The condition for the interior stationary point to exist under the state of emergency is given as shown in Lemma 2.

Lemma 2 The necessary and sufficient condition in order that $\hat{x}_{1} \in(0,1)$ under the state of emergency is given by

$$
(1+\rho) \sigma \zeta_{0}<u_{\text {out }}-u_{\text {home }}<\eta c+(1+\rho) \sigma\left(\zeta_{0}-\zeta_{1}\right) .
$$




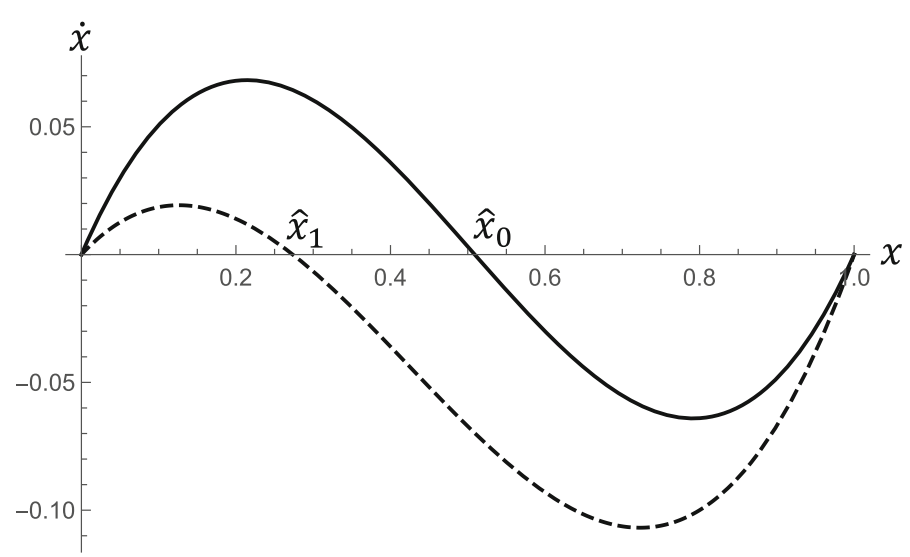

Fig. 2 Effect of the state of emergency. Notes: The figure shows the numerical plot of $\left.\dot{x}\right|_{\iota=0}$ drawn by solid line and $\left.\dot{x}\right|_{\iota=1}$ drawn by dash line with parameters as follows: $u_{\text {out }}=1, u_{\text {home }}=0.05, \eta=1, c=1.5$, $\sigma=0.5, \zeta_{0}=0.5, \zeta_{1}=0.25, \rho=1.5$

\section{Proof See "Appendix."}

Lemma 2 shows that the conditions for the existence of the interior stationary point under the non-legally binding state of emergency is similar to that in 1 .

The stability analysis at the stationary points under the state of emergency presents the following results:

Proposition 2 Under the state of emergency, the interior stationary point $x^{*}=\hat{x}_{1}$ is asymptotically stable and $x^{*} \in\{0,1\}$ is unstable under the following condition:

$$
\eta c>(1+\rho) \sigma \zeta_{1}
$$

Proof See "Appendix."

Proposition 2 shows that the interior stationary point is asymptotically stable and other stationary points are unstable, although there are three stationary points, $x^{*}=0, \hat{x}_{1}, 1$, as in Proposition 1. From Propositions 1 and 2, we need to compare each interior stationary point to consider the effect of the non-legal policy as the state of emergency.

We obtain the following proposition about the effects of the state of emergency.

Proposition 3 The state of emergency, which is a non-legally binding policy, has the effect of restraining the player's going-out behavior, that is, $\hat{x}_{1}-\hat{x}_{0}<0$, under the following condition:

$$
u_{\text {out }}-u_{\text {home }}<\frac{\zeta_{0}}{\zeta_{1}} \eta c
$$

Proof See "Appendix."

Proposition 3 suggests that a declaration of a state of emergency that is not legally binding discourages people from going out, which is consistent with the results in Japan that the number of people who go out reduces significantly under a state of emergency.

Figure 2 shows the numerical plot of the evolution of $x$ with and without the non-legally binding state of emergency. The stable interior stationary point uniquely exists in each evolution. We can visually confirm that $\hat{x}_{1}$ is lower than $\hat{x}_{0}$, that is, the non-legally binding state of emergency can reduce the share of going-out players through self-restraint behavior. 
The condition (C.3) in Proposition 3 means that the state of emergency is effective when the gain from going out is low, fixed stigma cost is high, degree of stigma reduction in players going out is higher, cost of infection is high, and the degree of increase in the subjective probability of infection of more players going out is high.

\section{Comparative Static Analysis}

We conduct a comparative static analysis to investigate the impact of varying each parameter ( $u_{\text {out }}, u_{\text {home }}, \eta, c, \sigma, \rho, \zeta_{0}$, and $\zeta_{1}$ ) on the equilibrium number of players going out. We summarize the results in the following proposition:

Proposition 4 Results in the comparative static analysis are given as follows:

1. An increase in the utility from going out $\left(u_{\text {out }}\right)$ raises the equilibrium share of players going out in the total population under the state of emergency and without it.

2. An increase in the utility from staying home $\left(u_{\mathrm{home}}\right)$ reduces the equilibrium share of players going out in the total population under the state of emergency and without it.

3. An increase in the degree of rise in the subjective probability of infection of more people going out $(\eta)$ reduces the equilibrium share of players going out in the total population under the state of emergency and without it.

4. An increase in the cost of infection (c) reduces the equilibrium share of players going out in the total population under the state of emergency and without it.

5. An increase in the relative size of stigma $(\sigma)$ reduces the equilibrium share of players going out in the total population under the state of emergency and without it.

6. An increase in the degree of stigma amplified by the state of emergency $(\rho)$ reduces the equilibrium share of players going out in the total population under the state of emergency.

7. An increase in the fixed stigma cost $\left(\zeta_{0}\right)$ reduces the equilibrium share of players going out in the total population under the state of emergency and without it.

8. An increase in the degree of stigma reduction of more people going out $\left(\zeta_{1}\right)$ raises the equilibrium share of players going out in the total population under the state of emergency and without it.

Proof See "Appendix."

Most of the results of Proposition 4 are consistent with our supposition. An increase in the utility from going out $\left(u_{\text {out }}\right)$ and the degree of stigma reduction in more people going out $\left(\zeta_{1}\right)$ raise the number of players going out because the incentive to go out increases.

In contrast, an increase in the utility from staying home $\left(u_{\text {home }}\right)$, degree of increase in the subjective probability of infection of more people going out $(\eta)$, cost of infection $(c)$, the relative size of stigma $(\sigma)$, the degree of stigma amplified by the state of emergency $(\rho)$, and the fixed stigma cost $\left(\zeta_{0}\right)$, reduce the number of players going out, because the incentive to go out decreases. 


\section{Welfare Analysis}

We now conduct the welfare analysis. Let $W$ denote social welfare, which is given by

$$
\begin{aligned}
W & =x E_{[G]}+(1-x) E_{[S]}, \\
& =x\left[u_{\text {out }}-\gamma(x) c-(1+\iota \rho) \sigma s(x)\right]+(1-x) u_{\text {home }}, \\
& =x\left[u_{\text {out }}-\eta c x-(1+\iota \rho) \sigma\left(\zeta_{0}-\zeta_{1} x\right)\right]+(1-x) u_{\text {home }} .
\end{aligned}
$$

Let $x^{\text {opt }}$ denote the socially optimal level of population share of players going out. The following proposition presents the relationship between the equilibrium level and the socially optimal level of $x$ :

Proposition 5 The interior equilibrium level of the population share of players going out is larger than the socially optimal level without/under the state of emergency, that is, $\hat{x}_{0}>x_{0}^{\mathrm{opt}}$, $\hat{x}_{1}>x_{1}^{\text {opt }}$.

\section{Proof See "Appendix."}

Proposition 5 suggests that the level of population share of players going out in the interior equilibrium is larger than the socially optimal level without the state of emergency, and it is the same under the state of emergency. The existence of externality in the model generates these results. Infection risk is assumed to be the increasing function with respect to the population share of going-out players and stigma is assumed to be the decreasing function.

That is, an increase in the number of people going out creates a negative externality of higher risk of infection and a positive externality of weaker stigma. Because each player considers the externalities for individual level, the equilibrium population share of players going out is excessive compared to the socially optimal level.

\section{Conclusion}

This study analyzes the interaction between self-restraint behavior, infection risk and stigma against going out during a pandemic using replicator dynamics. Consequently, the population share of going-out players has three steady states, as follows: $x^{*}=0, \hat{x}, 1$; however, the interior stationary point, $\hat{x}$, is asymptotically stable (Proposition 1 ). We show that the nonlegally binding policy reduces the number of people going out in a steady state by intensifying stigma costs (Proposition 3). This result is consistent with the empirical result in Katafuchi et al. [21]. Finally, the welfare analysis shows that the number of players going out is larger than the socially optimal level without/under the state of emergency (Proposition 5).

This study does not take into account any self-restraint on the part of suppliers, such as restaurants. However, the "self-restraint police" stigmatized not only people outdoors, but also restaurants operating in a declared state of emergency. We will need to analyze such supply-side for going out, household restraint behavior, and changes in the number of people infected and the economy. Our model assumes that stigma cost and infection risk are linear functions with respect to the population share of people going out. We will give their functions a micro-foundation for future work.

Social stigma is essential in the fight against COVID-19 because it reduces the spread of infection through individual self-restraint behavior. However, we must be vigilant of the negative side of stigma or social pressures because, as history shows, extreme stigmatization can lead to discrimination, prejudice, and violence. 


\section{Appendix}

\section{A.1: Proof of Lemma 1}

Proof First, the condition for $\hat{x}$ is positive is given by

$$
\begin{aligned}
\hat{x} & >0, \\
\frac{u_{\text {out }}-u_{\text {home }}-\sigma \zeta_{0}}{\eta c-\sigma \zeta_{1}} & >0, \\
u_{\text {out }}-u_{\text {home }}-\sigma \zeta_{0} & >0,
\end{aligned}
$$

Hence,

$$
u_{\text {out }}-u_{\text {home }}>\sigma \zeta_{0}
$$

Second, the condition for $\hat{x}$ is less than 1 is given by

$$
\begin{aligned}
\hat{x} & <1, \\
\frac{u_{\text {out }}-u_{\text {home }}-\sigma \zeta_{0}}{\eta c-\sigma \zeta_{1}} & <1, \\
u_{\text {out }}-u_{\text {home }}-\sigma \zeta_{0} & <\eta c-\sigma \zeta_{1},
\end{aligned}
$$

Thus,

$$
u_{\text {out }}-u_{\text {home }}<\eta c+\sigma\left(\zeta_{0}-\zeta_{1}\right) .
$$

From Conditions (17) and (18), the necessary and sufficient condition in order that $\hat{x} \in(0,1)$ is given by

$$
\sigma \zeta_{0}<u_{\text {out }}-u_{\text {home }}<\eta c+\sigma\left(\zeta_{0}-\zeta_{1}\right)
$$

\section{A.2: Proof of Proposition 1}

Proof We use the linear approximation method to check the stability in the stationary point. Differentiating $\dot{x}$ with respect to $x$ yields the following result:

$$
\frac{d \dot{x}}{d x}=(1-2 x)\left[u_{\text {out }}-u_{\text {home }}-\gamma(x) c-\sigma s(x)\right]-x(1-x)\left[\gamma^{\prime}(x) c+\sigma s^{\prime}(x)\right] .
$$

First, we check the stability condition for $x^{*}=0$. Substituting $x^{*}=0$ into Eq. (19), we obtain the following results:

$$
\left.\frac{d \dot{x}}{d x}\right|_{x^{*}=0}=u_{\text {out }}-u_{\text {home }}-\sigma \zeta_{0} .
$$

Thus, the stationary point $x^{*}=0$ is asymptotically stable if $u_{\text {out }}-u_{\text {home }}<\sigma \zeta_{0}$ and is otherwise unstable.

Second, we check the stability condition for $x^{*}=\hat{x}$. Substituting $x^{*}=\hat{x}$ into Eq. (19), we obtain the following results:

$$
\left.\frac{d \dot{x}}{d x}\right|_{x^{*}=\hat{x}}=-\hat{x}(1-\hat{x})\left[\eta c-\sigma \zeta_{1}\right] .
$$


The sign of (21) is negative when $\eta c>\sigma \zeta_{1}$. Thus, the stationary point $x^{*}=x_{1}$ is asymptotically stable.

Third, we confirm the stability condition for $x^{*}=1$. Substituting $x^{*}=1$ into Eq. (19), we obtain the following results:

$$
\left.\frac{d \dot{x}}{d x}\right|_{x^{*}=1}=-\left[u_{\text {out }}-u_{\text {home }}-\eta c-\sigma\left(\zeta_{0}-\zeta_{1}\right)\right] .
$$

Hence, the stationary point $x^{*}=1$ is asymptotically stable if $u_{\text {out }}-u_{\text {home }}>\eta c+\sigma\left(\zeta_{0}-\right.$ $\left.\zeta_{1}\right)$ and unstable otherwise. Summing up the above stability conditions and Lemma 1 , we conclude that the interior steady state $x^{*}=\hat{x}$ is asymptotically stable and $x^{*} \in\{0,1\}$ are unstable if an interior steady state exists.

\section{A.3: Proof of Lemma 2}

Proof First, the condition for $\hat{x}$ is positive is given by

$$
\begin{aligned}
\hat{x}_{1} & >0, \\
\frac{u_{\text {out }}-u_{\text {home }}-(1+\rho) \sigma \zeta_{0}}{\eta c-(1+\rho) \sigma \zeta_{1}} & >0, \\
u_{\text {out }}-u_{\text {home }}-(1+\rho) \sigma \zeta_{0} & >0,
\end{aligned}
$$

Hence,

$$
u_{\text {out }}-u_{\text {home }}>(1+\rho) \sigma \zeta_{0} .
$$

Second, the condition for $\hat{x}$ is less than 1 is given by

$$
\begin{aligned}
\hat{x}_{1} & <1, \\
\frac{u_{\text {out }}-u_{\text {home }}-(1+\rho) \sigma \zeta_{0}}{\eta c-(1+\rho) \sigma \zeta_{1}} & <1, \\
u_{\text {out }}-u_{\text {home }}-(1+\rho) \sigma \zeta_{0} & <\eta c-(1+\rho) \sigma \zeta_{1},
\end{aligned}
$$

Thus,

$$
u_{\text {out }}-u_{\text {home }}<\eta c+(1+\rho) \sigma\left(\zeta_{0}-\zeta_{1}\right) .
$$

From Conditions (23) and (24), the necessary and sufficient condition to ensure that $\hat{x}_{1} \in$ $(0,1)$ is given by

$$
(1+\rho) \sigma \zeta_{0}<u_{\text {out }}-u_{\text {home }}<\eta c+(1+\rho) \sigma\left(\zeta_{0}-\zeta_{1}\right)
$$

\section{A.4: Proof of Proposition 2}

Proof We use the linear approximation method to investigate the stability at the stationary point. The replicator dynamics of the population share of players going out is given by

$$
\left.\dot{x}\right|_{\iota=1}=x(1-x)\left[u_{\text {out }}-u_{\text {home }}-\gamma(x) c-(1+\rho) \sigma s(x)\right] .
$$


Differentiating (25) with respect to $x$ yields the following result:

$$
\begin{aligned}
\frac{d \dot{x}}{d x}= & (1-2 x)\left[u_{\text {out }}-u_{\text {home }}-\gamma(x) c-(1+\rho) \sigma s(x)\right] \\
& -x(1-x)\left[\gamma^{\prime}(x) c+(1+\rho) \sigma s^{\prime}(x)\right] .
\end{aligned}
$$

First, we check the stability condition for $x^{*}=0$. Substituting $x^{*}=0$ into Eq. (26), we obtain the following results:

$$
\left.\frac{d \dot{x}}{d x}\right|_{x^{*}=0}=u_{\text {out }}-u_{\text {home }}-(1+\rho) \sigma \zeta_{0} .
$$

Thus, the stationary point $x^{*}=0$ is asymptotically stable if $u_{\text {out }}-u_{\text {home }}<(1+\rho) \sigma \zeta_{0}$ and is otherwise unstable.

Second, we check the stability condition for $x^{*}=\hat{x}_{1}$. Substituting $x^{*}=\hat{x}_{1}$ into Eq. (26), we obtain the following results:

$$
\left.\frac{d \dot{x}}{d x}\right|_{x^{*}=\hat{x}_{1}}=-\hat{x}_{1}\left(1-\hat{x}_{1}\right)\left[\eta c-(1+\rho) \sigma \zeta_{1}\right] .
$$

The sign of (28) is negative when $\eta c>(1+\rho) \sigma \zeta_{1}$. Thus, the stationary point $x^{*}=\hat{x}_{1}$ is asymptotically stable.

Third, we confirm the stability condition for $x^{*}=1$. Substituting $x^{*}=1$ into Eq. (26), we obtain the following results:

$$
\left.\frac{d \dot{x}}{d x}\right|_{x^{*}=1}=-\left[u_{\text {out }}-u_{\text {home }}-\eta c-(1+\rho) \sigma\left(\zeta_{0}-\zeta_{1}\right)\right] .
$$

Hence, the stationary point $x^{*}=1$ is asymptotically stable if $u_{\text {out }}-u_{\text {home }}>(1+\rho) \eta c+$ $\sigma\left(\zeta_{0}-\zeta_{1}\right)$ and unstable otherwise. By summing up the above stability conditions and Lemma 2 , we conclude that the interior steady state $x^{*}=\hat{x}_{1}$ is asymptotically stable and $x^{*} \in\{0,1\}$ are unstable if an interior steady state exists.

\section{A.5: Proof of Proposition 3}

Proof The difference between $\hat{x}_{1}$ and $\hat{x}_{0}$ is given as follows:

$$
\hat{x}_{1}-\hat{x}_{0}=\frac{u_{\mathrm{out}}-u_{\mathrm{home}}-(1+\rho) \sigma \zeta_{0}}{\eta c-(1+\rho) \sigma \zeta_{1}}-\frac{u_{\mathrm{out}}-u_{\mathrm{home}}-\sigma \zeta_{0}}{\eta c-\sigma \zeta_{1}},
$$

From (30), the condition for $\hat{x}_{1}-\hat{x}_{0}<0$ is given by

$$
u_{\text {out }}-u_{\text {home }}<\frac{\zeta_{0}}{\zeta_{1}} \eta c .
$$

\section{A.6: Proof of Proposition 4}

Proof 1. We investigate the effect of an increase in the utility from going out in the equilibrium. The effect on the equilibrium under the state of emergency is given as follows:

$$
\frac{\partial \hat{x}_{1}}{\partial u_{\text {out }}}=\frac{1}{\eta c-(1+\rho) \sigma \zeta_{1}}>0
$$


while the effect on the equilibrium without the state of emergency is given by

$$
\frac{\partial \hat{x}_{0}}{\partial u_{\text {out }}}=\frac{1}{\eta c-\sigma \zeta_{1}}>0
$$

2. The effect of an increase in the utility from staying home on the equilibrium under the state of emergency is given as follows:

$$
\frac{\partial \hat{x}_{1}}{\partial u_{\text {out }}}=-\frac{1}{\eta c-(1+\rho) \sigma \zeta_{1}}<0,
$$

while the effect on the equilibrium without the state of emergency is given by

$$
\frac{\partial \hat{x}_{0}}{\partial u_{\text {out }}}=-\frac{1}{\eta c-\sigma \zeta_{1}}<0 .
$$

3. The effect of an increase in the degree of rise in the subjective probability of infection of more people going out on the equilibrium under the state of emergency is given as follows:

$$
\frac{\partial \hat{x}_{1}}{\partial \eta}=-\frac{c\left[u_{\mathrm{out}}-u_{\mathrm{home}}-(1+\rho) \sigma \zeta_{0}\right]}{\left[\eta c-(1+\rho) \sigma \zeta_{1}\right]^{2}}<0,
$$

while the effect on the equilibrium without the state of emergency is given by

$$
\frac{\partial \hat{x}_{0}}{\partial \eta}=-\frac{c\left[u_{\mathrm{out}}-u_{\mathrm{home}}-\sigma \zeta_{0}\right]}{\left(\eta c-\sigma \zeta_{1}\right)^{2}}<0
$$

4. The effect of an increase in the cost of infection on the equilibrium under the state of emergency is given as follows:

$$
\frac{\partial \hat{x}_{1}}{\partial \eta}=-\frac{\eta\left[u_{\text {out }}-u_{\text {home }}-(1+\rho) \sigma \zeta_{0}\right]}{\left[\eta c-(1+\rho) \sigma \zeta_{1}\right]^{2}}<0,
$$

while the effect on the equilibrium without the state of emergency is given by

$$
\frac{\partial \hat{x}_{0}}{\partial \eta}=-\frac{\eta\left[u_{\text {out }}-u_{\text {home }}-\sigma \zeta_{0}\right]}{\left(\eta c-\sigma \zeta_{1}\right)^{2}}<0
$$

5. The effect of an increase in the relative size of stigma on the equilibrium under the state of emergency is given as follows:

$$
\frac{\partial \hat{x}_{1}}{\partial \sigma}=-\frac{(1+\rho) \zeta_{0}}{\eta c-(1+\rho) \sigma \zeta_{1}}+\frac{\left[u_{\mathrm{out}}-u_{\mathrm{home}}-(1+\rho) \sigma \zeta_{0}\right](1+\rho) \zeta_{1}}{\left[\eta c-(1+\rho) \sigma \zeta_{1}\right]^{2}}<0,
$$

while the effect on the equilibrium without the state of emergency is given by

$$
\frac{\partial \hat{x}_{0}}{\partial \sigma}=-\frac{\zeta_{0}}{\eta c-\sigma \zeta_{1}}+\frac{\left(u_{\text {out }}-u_{\text {home }}-\sigma \zeta_{0}\right) \zeta_{1}}{\left(\eta c-\sigma \zeta_{1}\right)^{2}}<0
$$

6. The effect of an increase in the degree of stigma amplified by the state of emergency on the equilibrium under the state of emergency is given as follows:

$$
\frac{\partial \hat{x}_{1}}{\partial \rho}=-\frac{\sigma \zeta_{0}}{\eta c-(1+\rho) \sigma \zeta_{1}}+\frac{\left[u_{\mathrm{out}}-u_{\mathrm{home}}-(1+\rho) \sigma \zeta_{0}\right] \sigma \zeta_{1}}{\left[\eta c-(1+\rho) \sigma \zeta_{1}\right]^{2}}<0,
$$

while the effect on the equilibrium without the state of emergency is given by

$$
\frac{\partial \hat{x}_{0}}{\partial \rho}=0 \text {. }
$$


7. The effect of an increase in the fixed stigma cost on the equilibrium under the state of emergency is given as follows:

$$
\frac{\partial \hat{x}_{1}}{\partial \zeta_{0}}=-\frac{(1+\rho) \sigma}{\eta c-(1+\rho) \sigma \zeta_{1}}<0,
$$

while the effect on the equilibrium without the state of emergency is given by

$$
\frac{\partial \hat{x}_{0}}{\partial \zeta_{0}}=-\frac{\sigma}{\eta c-\sigma \zeta_{1}}<0
$$

8. The effect of an increase in the degree of stigma reduction of more people going out on the equilibrium under the state of emergency is given as follows:

$$
\frac{\partial \hat{x}_{1}}{\partial \zeta_{1}}=\frac{(1+\rho) \sigma\left[u_{\text {out }}-u_{\text {home }}-(1+\rho) \sigma \zeta_{0}\right]}{\left[\eta c-(1+\rho) \sigma \zeta_{1}\right]^{2}}>0,
$$

while the effect on the equilibrium without the state of emergency is given by

$$
\frac{\partial \hat{x}_{0}}{\partial \zeta_{1}}=\frac{\sigma\left[u_{\mathrm{out}}-u_{\mathrm{home}}-\sigma \zeta_{0}\right]}{\left(\eta c-\sigma \zeta_{1}\right)^{2}}>0 .
$$

\section{A.7: Proof of Proposition 5}

Proof Substituting $\iota=0$ into Eq. (16), we obtain the following:

$$
\left.W(x)\right|_{\iota=0}=x\left[u_{\text {out }}-\eta c x-\sigma\left(\zeta_{0}-\zeta_{1} x\right)\right]+(1-x) u_{\text {home }} .
$$

The first-order condition and the second order condition are given by

$$
\begin{aligned}
\frac{\left.d W(x)\right|_{\iota=0}}{d x} & =u_{\text {out }}-u_{\text {home }}-\sigma \zeta_{0}+2\left(\sigma \zeta_{1}-\eta c\right) x, \\
\frac{\left.d^{2} W(x)\right|_{\iota=0}}{d x^{2}} & =2\left(\sigma \zeta_{1}-\eta c\right)<0 .
\end{aligned}
$$

The socially optimal level of population share of going-out players without the state of emergency is as follows:

$$
x_{0}^{\mathrm{opt}}=\frac{u_{\mathrm{out}}-u_{\mathrm{home}}-\sigma \zeta_{0}}{2\left[\eta c-\sigma \zeta_{1}\right]}<\frac{u_{\mathrm{out}}-u_{\mathrm{home}}-\sigma \zeta_{0}}{\eta c-\sigma \zeta_{1}}=\hat{x}_{0} .
$$

Next, substituting $\iota=1$ into Eq. (16), we obtain the following:

$$
\left.W(x)\right|_{\iota=1}=x\left[u_{\text {out }}-\eta c x-(1+\rho) \sigma\left(\zeta_{0}-\zeta_{1} x\right)\right]+(1-x) u_{\text {home }} .
$$

The first-order condition and the second-order condition are given by

$$
\begin{aligned}
\frac{\left.d W(x)\right|_{\iota=1}}{d x} & =u_{\text {out }}-u_{\text {home }}-(1+\rho) \sigma \zeta_{0}+2\left[(1+\rho) \sigma \zeta_{1}-\eta c\right] x, \\
\frac{\left.d^{2} W(x)\right|_{\iota=1}}{d x^{2}} & =2\left[(1+\rho) \sigma \zeta_{1}-\eta c\right] x<0 .
\end{aligned}
$$


The socially optimal level of population share of players going out under the state of emergency is as follows:

$$
x_{1}^{\mathrm{opt}}=\frac{u_{\mathrm{out}}-u_{\mathrm{home}}-(1+\rho) \sigma \zeta_{0}}{2\left[\eta c-(1+\rho) \sigma \zeta_{1}\right]}<\frac{u_{\mathrm{out}}-u_{\mathrm{home}}-(1+\rho) \sigma \zeta_{0}}{\eta c-(1+\rho) \sigma \zeta_{1}}=\hat{x}_{1} .
$$

\section{References}

1. Acemoglu D, Chernozhukov V, Werning I, Whinston MD (2020) A multi-risk sir model with optimally targeted lockdown. NBER working paper. https://www.nber.org/papers/w27102. Accessed 11 July 2020

2. Alger I, Weibull JW, Lehmann L (2020) Evolution of preferences in structured populations: genes, guns, and culture. J Econ Theory 185:104951

3. Alvarez FE, Argente D, Lippi F (2020) A simple planning problem for covid-19 lockdown. NBER working paper https://www.nber.org/papers/w26981. Accessed 11 July 2020

4. AS (2020) Coronavirus: Spain fines over 1 million lockdown dodgers. https://en.as.com/en/2020/05/20/ other_sports/1590007192_285496.html. Accessed 4 Oct 2020

5. Besley T, Coate S (1992) Understanding welfare stigma: taxpayer resentment and statistical discrimination. J Public Econ 48:165-183. https://doi.org/10.1016/0047-2727(92)90025-B

6. Bhargava S, Manoli D (2015) Psychological frictions and the incomplete take-up of social benefits: evidence from an IRS field experiment. Am Econ Rev 105(11):3489-3529. https://doi.org/10.1257/aer. 20121493

7. Bouveret G, Mandel A (2021) Social interactions and the prophylaxis of si epidemics on networks. J Math Econ 93:102486

8. Cerqueti R, Correani L, Garofalo G (2013) Economic interactions and social tolerance: a dynamic perspective. Econ Lett 120(3):458-463

9. COVID-19 Situazione Italia (2020) COVID-19 Italia. https://opendathunadpc.maps.arcgis.com/apps/ opsdashboard/index.html\#/b0c68bce2cce478eaac82fe38d4138b1. Accessed 4 Oct 2020

10. Eichenbaum MS, Rebelo S, Trabandt M (2020) The macroeconomics of testing and quarantining. NBER working paper https://www.nber.org/papers/w27104. Accessed 11 July 2020

11. Ennis HM, Weinberg JA (2013) Over-the-counter loans, adverse selection, and stigma in the interbank market. Rev Econ Dyn 16(4):601-616

12. Farboodi M, Jarosch G, Shimer R (2020) Internal and external effects of social distancing in a pandemic. NBER working paper. https://www.nber.org/papers/w27059. Accessed 11 July 2020

13. France 24 (2020) French National Assembly greenlights coronavirus 'public health emergency' bill. https://www.france24.com/en/20200322-coronavirus-french-national-assembly-greenlights-publichealth-emergency-bill. Accessed 4 Oct 2020

14. García J, van den Bergh JC (2011) Evolution of parochial altruism by multilevel selection. Evol Hum Behav 32(4):277-287

15. Gharehgozli O, Nayebvali P, Gharehgozli A, Zamanian Z (2020) Impact of COVID-19 on the economic output of the US outbreak's epicenter. Econ Disast Clim Change. https://doi.org/10.1007/s41885-02000069-w

16. Goffman E (1963) Stigma: notes on the management of spoiled identity. Prentice-Hall, Upper Saddle River

17. Henrich J (2009) The evolution of costly displays, cooperation and religion: credibility enhancing displays and their implications for cultural evolution. Evol Hum Behav 30(4):244-260

18. Holtemöller O (2020) Integrated assessment of epidemic and economic dynamics. IWH discussion papers. https://www.econstor.eu/bitstream/10419/215895/1/1694465632.pdf. Accessed 11 July 2020

19. Itaya J, Kurita K (2020) Replicator evolution of welfare stigma: welfare fraud vs incomplete takeup. CESifo working paper, pp 1-29. https://www.cesifo.org/en/publikationen/2020/working-paper/ replicator-evolution-welfare-stigma-welfare-fraud-vs-incomplete. Accessed 5 Nov 52020

20. Iwasaki A, Grubaugh ND (2020) Why does japan have so few cases of covid-19? EMBO Mol Med 12(5):e12481

21. Katafuchi Y, Kurita K, Managi S (2021) Covid-19 with stigma: theory and evidence from mobility data. Econ Disast Clim Change 5(1):71-95

22. Kim Y (2003) Income distribution and equilibrium multiplicity in a stigma-based model of tax evasion. J Public Econ 87(7-8):1591-1616 
23. Kobayashi G, Sugasawa S, Tamae H, Ozu T (2020) Predicting intervention effect for covid-19 in Japan: state space modeling approach. BioScience Trends

24. Kurita K, Hori N, Katafuchi Y (2020) Stigma model of welfare fraud and non-take-up: theory and evidence from OECD panel data. Int J Econ Theory, pp 1-29

25. Kyodo News (2020) Morning crowds down 60\% in central Tokyo after emergency declared. https:// english.kyodonews.net/news/2020/04/4394741a8810-morning-crowds-down-60-in-central-tokyoafter-emergency-declared.html. Accessed 11 July 2020

26. Lieberman DL, Tybur JM, Latner JD (2012) Disgust sensitivity, obesity stigma, and gender: contamination psychology predicts weight bias for women, not men. Obesity 20(9):1803-1814

27. Lindbeck A, Nyberg S, Weibull JW (1999) Social norms and economic incentives in the welfare state. Q J Econ 114(1):1-35

28. Lu N, Cheng KW, Qamar N, Huang KC, Johnson JA (2020) Weathering covid-19 storm: successful control measures of five Asian countries. Am J Infect Control 48(7):851-852

29. Major B, Dovidio JF, Link BG (2018) The Oxford handbook of stigma, discrimination, and health. Oxford University Press. http://search.ebscohost.com/login.aspx?direct=true\&db=edsnop\&AN=edsnop. I028539219.00\&site=eds-live

30. Mandel A, Veetil V (2020) The economic cost of COVID lockdowns: an out-of-equilibrium analysis. Econ Disast Clim Change. https://doi.org/10.1007/s41885-020-00066-z

31. Martin A, Markhvida M, Hallegatte S, Walsh B (2020) Socio-economic impacts of COVID-19 on household consumption and poverty. Econ Disast Clim Change. https://doi.org/10.1007/s41885-020-000703

32. Moffitt R (1983) An economic model of welfare stigma. Am Econ Rev 73(5):1023-1035

33. Norman TW (2020) The evolution of monetary equilibrium. Games and Economic Behavior

34. Rasmusen E (1996) Stigma and self-fulfilling expectations of criminality. J Law Econ 39(2):519-543

35. Safarzyńska K, Van den Bergh JC (2011) Beyond replicator dynamics: innovation-selection dynamics and optimal diversity. J Econ Behav Org 78(3):229-245

36. Shi Y, Pan M, Peng D (2017) Replicator dynamics and evolutionary game of social tolerance: the role of neutral agents. Econ Lett 159:10-14

37. Taylor PD, Jonker LB (1978) Evolutionary stable strategies and game dynamics. Math Biosci 40(12):145-156

38. The COVID Tracking Project (2020) Our DATA. https://covidtracking.com/data. Accessed 4 Oct 2020

39. The Japan Times (2020) Japan cautiously lifts last of virus emergency controls. https://www.japantimes. co.jp/news/2020/05/25/national/japan-lifts-state-of-emergency-coronavirus/. Accessed 11 July 2020

40. The Japan Times (2020) Japan's 'virus vigilantes' take on rule-breakers and invaders. https://www. japantimes.co.jp/news/2020/05/13/national/coronavirus-vigilantes-japan/. Accessed 11 July 2020

41. The Japan Times (2020c) Understanding the need to shame someone on social media for not exercising self-restraint during a pandemic. https://www.japantimes.co.jp/news/2020/05/16/national/medianational/social-media-shaming-coronavirus/. Accessed 11 July 2020

42. Wilson DS, O'Brien DT, Sesma A (2009) Human prosociality from an evolutionary perspective: variation and correlations at a city-wide scale. Evol Hum Behav 30(3):190-200

43. Wood AD, Mason CF, Finnoff D (2016) Opec, the seven sisters, and oil market dominance: an evolutionary game theory and agent-based modeling approach. J Econ Behav Org 132:66-78

44. Wu J (2018) Entitlement to assort: democracy, compromise culture and economic stability. Econ Lett $163: 146-148$

45. Wu J (2019) Labelling, homophily and preference evolution. Int J Game Theory, 1-22

46. Yamamura E, Tsutsui Y (2020) Impact of the state of emergency declaration for covid-19 on preventive behaviors and mental conditions in Japan: difference in difference analysis using panel data. arXiv preprint arXiv:2005.13008

47. Yang Z, Shi Y, Li Y (2018) Analysis of intellectual property cooperation behavior and its simulation under two types of scenarios using evolutionary game theory. Comput Ind Eng 125:739-750

Publisher's Note Springer Nature remains neutral with regard to jurisdictional claims in published maps and institutional affiliations. 